\title{
An agonistic monoclonal antibody against DR5 induces ROS production, sustained JNK activation and Endo G release in Jurkat leukemia cells
}

\author{
Caifeng Chen", Yanxin Liu', Dexian Zheng ${ }^{1}$ \\ ${ }^{\prime}$ National Laboratory of Medical Molecular Biology, Institute of Basic Medical Sciences, Chinese Academy of Medical Sciences \& \\ Peking Union Medical College, 5 Dong Dan San Tiao, Beijing 100005, China
}

We have previously reported that AD5-10, a novel agonistic monoclonal antibody against DR5, possessed a strong cytotoxic activity in various tumor cells, via induction of caspase-dependent and -independent signaling pathways. The present study further demonstrates that reactive oxygen species (ROS) were generated in abundance in Jurkat leukemia cells upon AD5-10 stimulation and that ROS accumulation subsequently evoked sustained activation of c-Jun N-terminal kinase (JNK), loss of mitochondrial membrane potential, and release of endonuclease G (Endo G) from mitochondria into the cytosol. The reducing agent, $\mathrm{N}$-acetylcysteine (NAC), effectively inhibited the sustained activation of JNK, release of Endo G, and cell death in Jurkat cells treated by AD5-10. Moreover, a dominant-negative form of JNK (but not of p38) enhanced NF- $\mathrm{kB}$ activation, suppressed caspase-8 recruitment in death-inducing signaling complexes (DISCs), and reduced adverse effects on mitochondria, thereby inhibiting AD5-10-induced cell death in Jurkat leukemia cells. These data provide novel information on the DR5-mediated cell death-signaling pathway and may shed new light on effective strategies for leukemia and solid tumor therapies.

Keywords: AD5-10, ROS, JNK, DR5, TRAIL

Cell Research (2009) 19:984-995. doi: 10.1038/cr.2009.60; published online 26 May 2009

\section{Introduction}

Tumor necrosis factor related apoptosis-inducing ligand (TRAIL), a member of the tumor necrosis factor (TNF) superfamily, selectively induces cell death in cancer cells without cytotoxicity to most normal cells either in vitro or in vivo. These are two functional receptors for

Correspondence: Dexian Zheng, Yanxin Liu

Tel: +86-10-6529 6409; Fax: +86-10-6510 5102

E-mail: zhengdx@pumc.edu.cn or zhengdx@tom.com

Abbreviations: TRAIL (tumor necrosis factor-related apoptosis-inducing ligand); PCD (programmed cell death); DD (death domain); TNF (tumor necrosis factor); FasL (Fas ligand); TRAIL-R (TRAIL receptor); DR (death receptor); DcR (decoy receptor); FADD (Fas-associated death domain protein); JNK (c-Jun N-terminal kinase); RIP (receptor-interacting protein); PARP (poly (ADP-ribose) polymerase); AIF (apoptosis-inducing factor); Endo G (endonuclease G); PBS (phosphate buffered saline); FCS (fetal calf serum); MEFs (murine embryonic fibroblasts); ROS (reactive oxygen species); NAC (N-acetylcysteine); GSH (glutathione); NAO (nonyl-acridine Orange)

Received 30 December 2008; revised 10 April 2009; accepted 7 May 2009; published online 26 May 2009
TRAIL, DR4 (TRAIL-R1) and DR5 (TRAIL-R2), each containing a cytoplasmic death domain (DD), which triggers cell death upon TRAIL binding. Three decoy receptors, DcR1 (DcR1/TRAIL-R3), DcR2 (TRAIL-R4), and osteoprotegerin, block TRAIL-induced cell death $[1,2]$. Binding of TRAIL to DR4 or DR5 leads to trimerization of the receptor, resulting in the recruitment of Fasassociated death domain protein (FADD), caspase-8, and receptor-interacting protein (RIP) to form the deathinducing signaling complex (DISC) $[3,4]$. DISCs initiate a caspase cascade by activating apical caspase- 8 , which cleaves and activates the downstream caspases 3,6 , and/ or 7, leading to cell death [4]. Hence, agonistic monoclonal antibodies (mAbs) specific for human DR4 or DR5, which selectively kill tumor cells, have become attractive candidates for cancer therapy.

Apart from the classical caspase cascade, c-Jun Nterminal kinase (JNK) and p38 MAP kinase are often activated in cell death [5]. As sensors of environmental stress, activation of JNK and p38 can be triggered by many factors, especially chemotherapeutic agents, ul- 
traviolet radiation, and ischemia $[5,6]$. Cytokines of the TNF superfamily are also important inducing signals for JNK and p38 activation and even cell death [7, 8]. Previous studies have shown that TNF $\alpha$ or Fas ligand (FasL) can induce instantaneous activation of JNK within 20 min and subsequently sustain JNK activation [9-13]. p38 was also activated in cells treated with $\mathrm{TNF} \alpha$ or FasL [11]. Sakon et al. [10] and Kamata et al. [9] have demonstrated that $\mathrm{TNF} \alpha$-induced generation of reactive oxygen species (ROS) sustained JNK and p38 activation, respectively. Ventura et al. [14] have provided evidence that JNK is able to potentiate TNF $\alpha$-induced production of ROS. Taken together, these results suggest that JNK and ROS work together in a positive feedback loop to accelerate programmed cell death. However, there is still no detailed information on DR4- or DR5-mediated activation of JNK or p38. How is JNK or p38 activated by TRAIL or agonistic mAbs? Does the activation of JNK or p38 directly augment cell death mediated by DR4/ DR5? These questions remain to be satisfactorily answered. Various chemotherapeutic agents were combined with TRAIL in previous studies [15-17], despite the fact that chemotherapeutic agents have complicated effects on cell survival and death. Many chemotherapeutic agents are reported to enhance TRAIL-induced apoptosis in tumor cells via ROS production and mitochondrial dysfunction [18-22].

In our previous study, we reported a novel anti-human DR5 monoclonal antibody, AD5-10, with strong cytotoxic effects on various tumor cells. In the present study, we further investigated the molecular mechanisms of the signaling pathway initiated by AD5-10. We demonstrate that AD5-10-induced cell death in Jurkat cells occurs via ROS generation, sustained JNK activation, loss of mitochondrial membrane potential, and Endo G release from mitochondria to the cytosol.

\section{Results}

AD5-10 induces ROS generation and GSH level reduction in Jurkat leukemia cells

Oxidative stress is often closely associated with cell
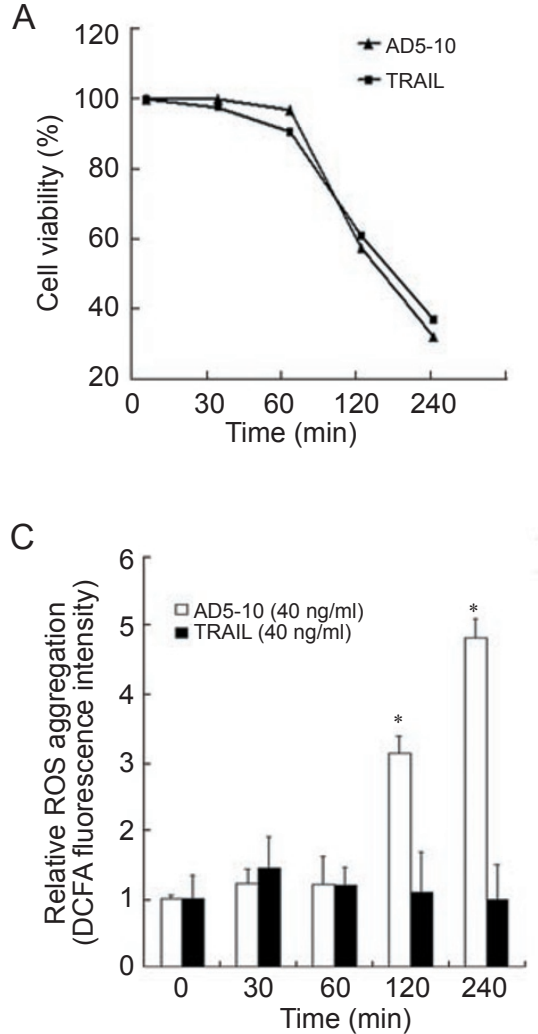

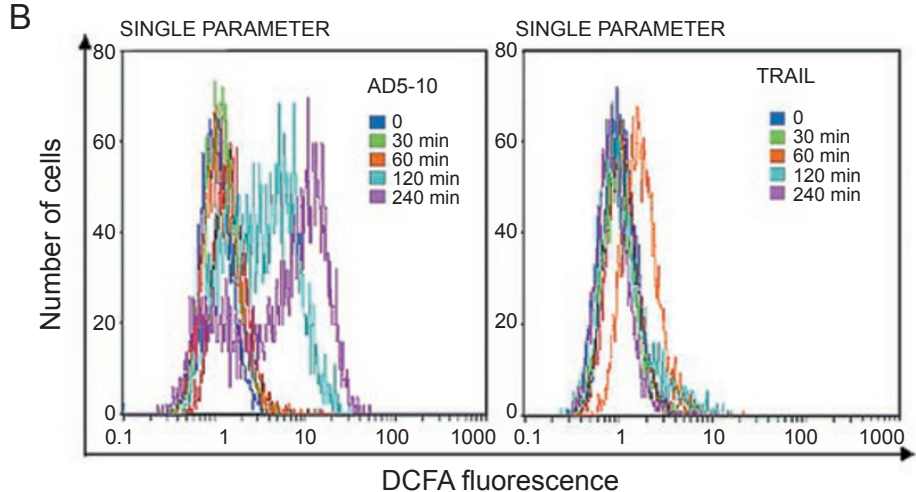

D

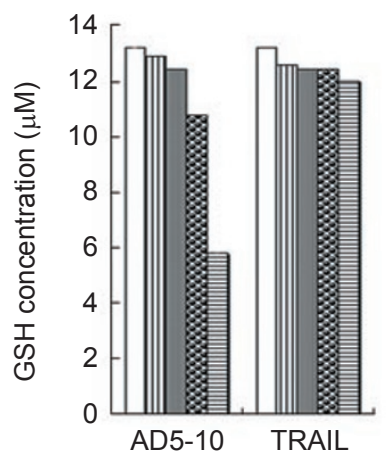

$\square 0$ m $30 \mathrm{~min}$ $\square 60 \mathrm{~min}$ (ख) $120 \mathrm{~min}$ 目 $240 \mathrm{~min}$

Figure 1 AD5-10 induces ROS production and reduces GSH level in Jurkat cells. Cells were treated with AD5-10 (40 ng/ml) or TRAIL $(40 \mathrm{ng} / \mathrm{ml})$ or left untreated for the indicated timepoints. Cell viability was detected by MTS assay (A), ROS production was detected with DCFA2 fluorescence (B) and quantitated (C), and GSH and GSSG levels were analyzed (D). Error bars represent the standard deviation from the mean value obtained from triplicate. Data are representative of three independent experiments. 
death. Overproduction of reactive oxygen species (ROS) and reduction of GSH level are considered to promote cell death in response to a variety of signals and in various pathophysiological settings [14, 23]. Jurkat cells, which highly express DR5, but express few DR4 molecules [24], were shown to be senstive to TRAIL and AD5-10 in our previous report [2]. To investigate whether the change of cellular redox state is involved in DR5mediated cell signaling, Jurkat cells were treated with AD5-10 (40 ng/ml) or TRAIL $(40 \mathrm{ng} / \mathrm{ml})$ for various timepoints. Cell viability was detected by the MTS assay, and ROS generation was measured by $4 \mu \mathrm{M} 2^{\prime}, 7^{\prime}$-dichlorofluorescin-diacetate (DCFA) staining, followed by flow cytometry. DCFA is a redox sensitive dye that can be cleaved by intracellular esterases in the presence of ROS and then converted into the fluorescent species $2^{\prime}, 7^{\prime}$-dichlorfluorescin (DCF). DCF fluorescence thus represents the generation of ROS. The ratio of reduced glutathione (GSH) to oxidized glutathione (GSSG) was detected by a GSH/GSSG assay kit. Both AD5-10 and TRAIL reduced cell viability in Jurkat cells in a time-dependent manner (Figure 1A). The DCF fluorescence intensity in AD510 -treated cells was dramatically amplified over the time course (Figure 1B), indicating that there was a sixfold increase in ROS generation in AD5-10-treated Jurkat cells within $4 \mathrm{~h}$ (Figure 1C). However, there was no significant ROS production in TRAIL-treated cells (Figure $1 \mathrm{~B}$ and $1 \mathrm{C})$. Correspondingly, GSH and GSSG content in AD5-10-treated Jurkat cells showed a time-dependent reduction, but no changes were observed in the cells
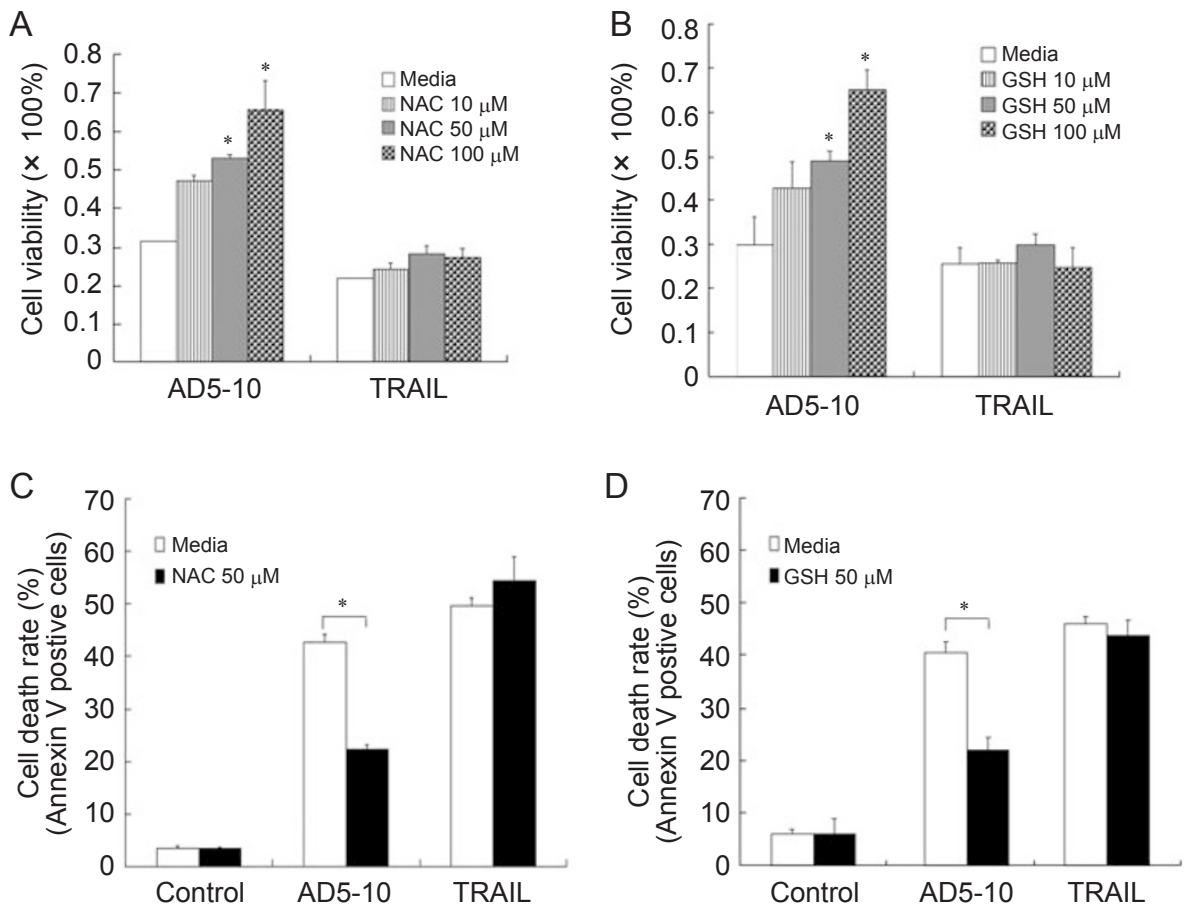

E

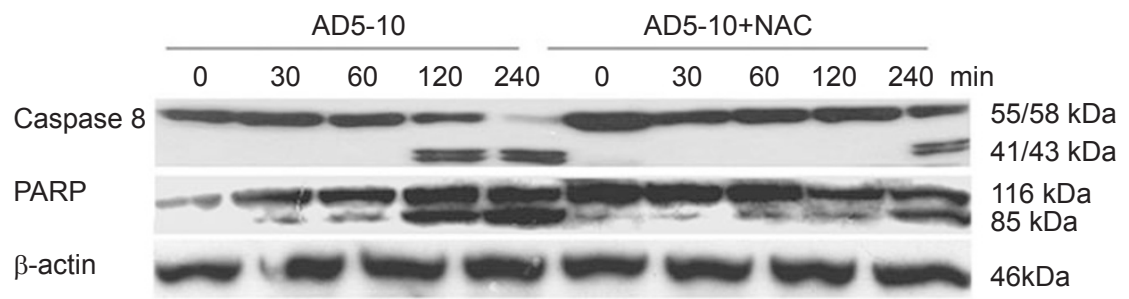

Figure 2 NAC and GSH inhibit AD5-10-induced cell death in Jurkat cells. Cells were treated with AD5-10 (40 ng/ml) or TRAIL $(40 \mathrm{ng} / \mathrm{ml})$ in the absence or presence of indicated concentration of NAC (A, C) or GSH (B, D) for 4 h. Cell viability (A, B) was detected by MTS assay, and apoptosis (C, D) was analyzed by Annexin-V-FITC and PI staining, followed by flow cytometry. (E) Cells treated with AD5-10 (40 ng/ml) or left untreated in the absence or presence of $50 \mu \mathrm{M}$ NAC for the indicated timepoints were used for western blot analysis of caspase- 8 and PARP cleavage with the indicated specific antibodies. Error bars represent the standard deviation from the mean value obtained from triplicate. Data are representative of three independent experiments. 
treated with TRAIL (Figure 1D). These data demonstrate that AD5-10 specifically induces ROS generation and GSH level reduction in Jurkat leukemia cells.

\section{NAC inhibits AD5-10-induced cell death}

To further evaluate the role of redox state in AD5-10induced cell death, Jurkat cells were treated with AD5-10 $(40 \mathrm{ng} / \mathrm{ml})$ or TRAIL $(40 \mathrm{ng} / \mathrm{ml})$ or left untreated for $4 \mathrm{~h}$ in the presence or absence of N-acetyl-L-cysteine (NAC) or reduced glutathione (GSH), which are classical thiol antioxidants capable of acting as ROS scavengers. Cell viability was detected by the MTS assay, and cell death was detected by Annexin-V/PI staining followed by flow cytometry. As shown in Figure 2, in the presence of NAC (Figure 2A) or GSH (Figure 2B), cell viability of AD5-10-treated cells was increased in a dose-dependent manner, but no such effect was observed in cells treated with TRAIL. Flow cytometry analysis showed that about $45 \%$ of Jurkat cells treated with AD5-10 were Annexin-V-positive, but that only approximately $22 \%$ were Annexin-V-positive when treated with AD5-10 in the presence of $50 \mu \mathrm{M}$ NAC (Figure 2C) or GSH (Figure 2D). Signaling pathway analysis by western blot showed that pro-caspase- 8 and its substrate PARP were cleaved in cells treated with AD5-10 for 2 and $4 \mathrm{~h}$ and that NAC pretreatment efficiently inhibited their cleavage (Figure 2E). These data confirm that NAC markedly reduces death in Jurkat cells treated with AD5-10, suggesting that ROS and redox state have significant effects on the DR5mediated cell death pathway.

\section{NAC inhibits AD5-10-induced mitochondrial dysfunction}

Mitochondria are a dynamic organelle sensitive to various stimuli. They are both the source and the target of ROS, and the buildup of ROS is thought to greatly impair their normal function. To clarify whether mitochondrial function is affected by AD5-10-induced ROS production, Jurkat cells were treated with AD5-10 or left untreated for $60 \mathrm{~min}, 120 \mathrm{~min}$, and $240 \mathrm{~min}$ in the presence or absence of $50 \mu \mathrm{M}$ NAC. Mitochondrial membrane potential was detected by $\mathrm{JC}-1$, and oxidation of cardiolipin was evaluated with nonyl-acridine orange (NAO) staining followed by flow cytometry. JC-1 accumulates in mitochondria as aggregates, resulting in red fluorescence in non-apoptotic cells. The monomeric form, which fluoresces green, is found in apoptotic as well as necrotic cells. NAO is used to detect cardiolipin, which loses the ability to bind NAO when oxidized by ROS. As shown in Figure 3A and 3B, the green fluorescence of JC-1 increased, and the mass of primary cardiolipin was reduced significantly in Jurkat cells treated with AD5-10 over the time course. These results indicate that mitochondrial membrane potential decreased and cardiolipin was oxidized. NAC treatment effectively blocked cardiolipin oxidation and the reduction in mitochondrial membrane potential in Jurkat cells treated with AD5-10. Bcl-2 family proteins are also critical factors in apoptosis, especially in cell death associated with oxidative stress [25]. As shown by western blotting in Figure $3 \mathrm{C}$, Bid was cleaved in cells treated with AD5-10 in a time-dependent manner, whereas the cellular expression of other family members, such as Bcl-2, Bcl-xl, Bik, and $\mathrm{Bad}$, did not change. Again, NAC pretreatment effectively inhibited the cleavage of Bid induced by AD5-10.

Loss of mitochondrial membrane potential, oxidative stress, and Bid cleavage may severely damage mitochondria, leading to the release of pro-apoptotic factors, including cytochrome c (Cyt c), endonuclease G (Endo G), and apoptosis inducing factor (AIF) [25-27] into the cytosol. Cyt $\mathrm{c}$ release results in the activation of caspase- 9 [26], and the release of Endo G and AIF is involved in a caspase-independent death pathway $[27,28]$. To evaluate the involvement of the release of pro-apoptotic factors from mitochondria in DR5-mediated cell death, Jurkat cells were treated with AD5-10 $(40 \mathrm{ng} / \mathrm{ml})$ or TRAIL $(40 \mathrm{ng} / \mathrm{ml})$ or left untreated for $4 \mathrm{~h}$ in the presence or absence of $50 \mu \mathrm{M}$ NAC. Mitochondrial, cytosolic, and nuclear fractions were isolated and subjected to western blot analysis using specific antibodies. As shown in Figure 3D, NAC effectively inhibited the release of AIF and Endo G in the cells treated with AD5-10, whereas it had little effect in the cells treated with TRAIL. Furthermore, the increase of Endo $G$ in the nuclear fraction was suppressed by NAC in a dose-dependent manner, whereas the amount of nuclear AIF was not notably altered (Figure 3E).

To further demonstrate the localization of Endo G, Jurkat and HCT116 cells were treated with AD5$10(40 \mathrm{ng} / \mathrm{ml})$ or left untreated for $4 \mathrm{~h}$ in the presence or absence of $50 \mu \mathrm{M}$ NAC. Cells were then probed with 4',6-diamidino-2-phenylindole (DAPI) and FITClabeled anti-Endo $\mathrm{G}$ antibody, followed by confocal laser microscopy. As shown in Figure $3 \mathrm{Fa}$ and $3 \mathrm{Fj}$, Endo $\mathrm{G}$ (green) was normally localized in the cytoplasm around the nucleus (blue) in control cells (Figure 3Fb, c, k and l). After a 4-h treatment with AD5-10, Endo G levels in the cytoplasm were reduced (Figure 3Fd and 3Fm). Endo G appeared to be confined to the nucleus (Figure $3 \mathrm{Fe}, \mathrm{f}, \mathrm{n}$ and o), indicating that Endo G translocated to the nucleus upon stimulation with AD5-10. Moreover, pretreatment with $50 \mu \mathrm{M}$ NAC blocked the translocation of Endo $\mathrm{G}$ induced by AD5-10 (Figure 3Fg, h, i, p, q, and r).

These data indicate that AD5-10-induced ROS accumulation in Jurkat cells further impairs mitochondrial membrane potential, leading to oxidation of lipoproteins 
A

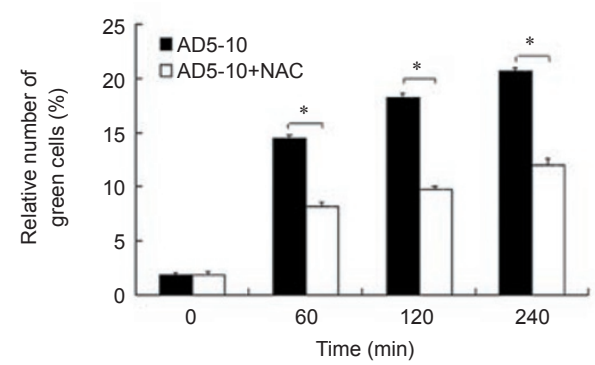

C

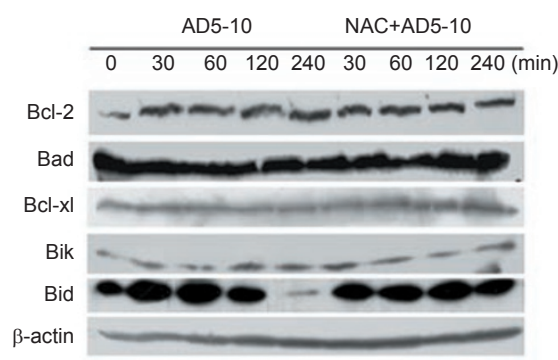

E

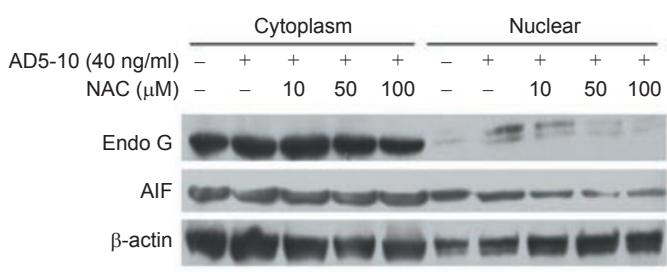

B
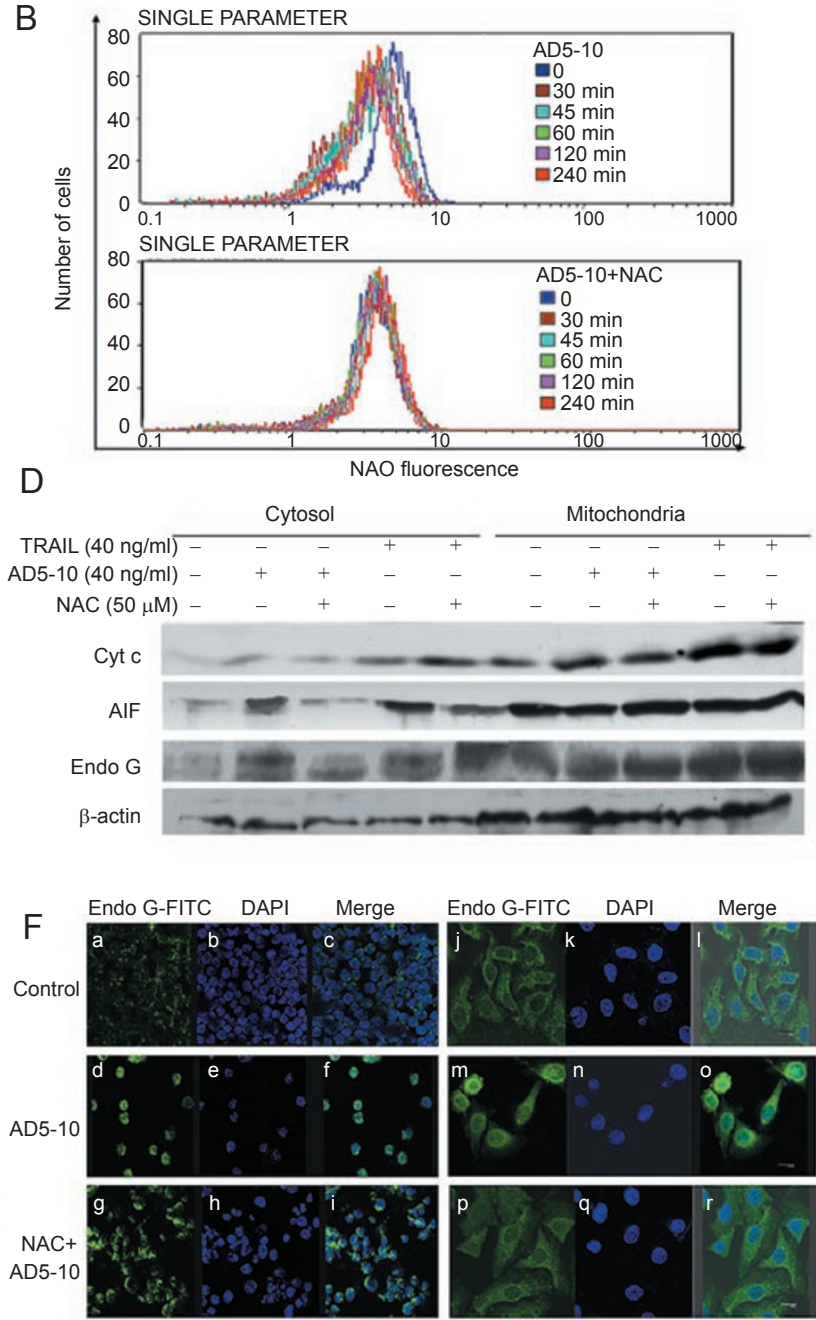

Figure 3 NAC inhibits AD5-10-induced mitochondrial dysfunction in Jurkat cells. Cells were treated with AD5-10 (40 ng/ml) or left untreated in the absence or presence of $50 \mu \mathrm{M}$ NAC for the indicated timepoints. Mitochondrial membrane potential $(\Delta \psi \mathrm{m})$ was determined with $\mathrm{JC}-1(40 \mathrm{nM})$ staining $(\mathrm{A})$, and oxidative damage to mitochondria was evaluated by $\mathrm{NAO}$ staining (B) followed by flow cytometry. Expression of $\mathrm{Bcl}-2$ family proteins was detected by western blot with specific antibodies (C). In addition, cells were treated with AD5-10 $(40 \mathrm{ng} / \mathrm{ml})$ or TRAIL $(40 \mathrm{ng} / \mathrm{ml})$ or left untreated in the absence or presence of $50 \mu \mathrm{M}$ NAC for $4 \mathrm{~h}$, and mitochondrial and cytosolic fractions were isolated and subjected to immunoblot analysis to determine the release of cytochrome c, Endo G and AIF (D). Meanwhile, nuclear and cytoplasmic fractions were isolated and subjected to immunoblot analysis to determine the expression level of Endo $G$ and AIF (E). Immunofluorescence imaging of Endo G translocation in Jurkat and HCT116 cells was observed by confocal laser microscope (F), in which cells were treated with AD5-10 $(40 \mathrm{ng} / \mathrm{ml})$ in the absence ( $\mathrm{d}, \mathrm{e}, \mathrm{f}, \mathrm{m}, \mathrm{n}$, and o) or presence $(\mathrm{g}, \mathrm{h}, \mathrm{l}, \mathrm{p}, \mathrm{q}$, and r) of $50 \mu \mathrm{M} \mathrm{NAC}$ for $4 \mathrm{~h}$ and stained with DAPI (b, e, h, k, n, and q) or antibodies against Endo G (a, d, g, j, m, and p). Untreated cells were used as controls (a, b, c, j, k, and l). Images from each staining were also merged (c, f, l, I, o, and r). Error bars represent the standard deviation from the mean value obtained from triplicate. Data are representative of three independent experiments.

and Endo G translocation. All of these effects are suppressed by NAC.

\section{NAC inhibits AD5-10-induced sustained activation of JNK}

$\mathrm{TNF} \alpha$ or FasL can activate JNK and p38 in various cells [10-14]. These factors are closely associated with necrotic cell death [6-9]. ROS have also been identi- fied as major mediators of TNF-induced JNK activation in murine embryonic fibroblasts (MEFs) [11, 14]. To investigate the role of JNK and p38 activation in AD5-10-induced cell death, Jurkat cells were treated as described above, and JNK and p38 activation was detected by western blot analysis. As shown in Figure 4A, phosphorylated JNK (p-JNK) levels were transiently 
A

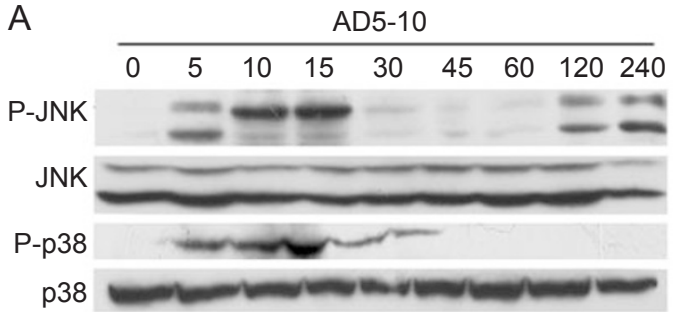

B

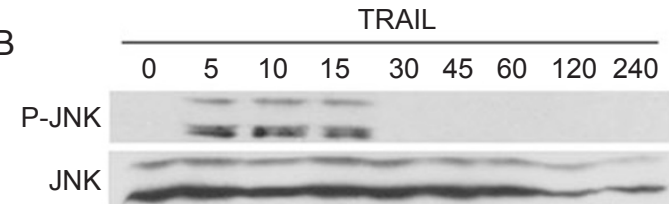

$N A C+A D 5-10$

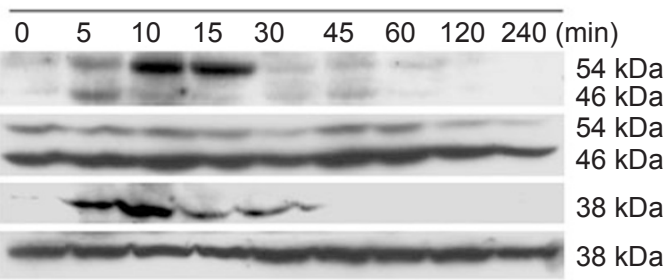

NAC+TRAIL

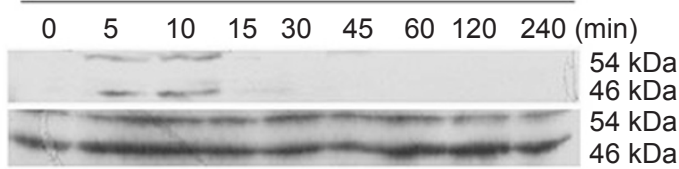

Figure 4 NAC inhibits AD5-10-induced sustained JNK activation in Jurkat cells. Cells were incubated with AD5-10 (40 ng/ml) (A) or TRAIL $(40 \mathrm{ng} / \mathrm{ml})(B)$ in the absence or presence of $50 \mu \mathrm{M}$ NAC for the indicated timepoints. Phosphorylation of JNK and p38 was detected by western blot assay using specific antibodies.

increased in the cells treated with AD5-10 at 5 to $15 \mathrm{~min}$, were undetectable at 30 and $60 \mathrm{~min}$, and were detectable again from 2 to $4 \mathrm{~h}$. These results suggest that sustained activation of JNK was induced by AD5-10, whereas no sustained activation of p38 (in the form of phospho-p38) was observed. It is very interesting to note that NAC pretreatment completely abolished AD5-10-induced sustained JNK activation, but not transient JNK or p38 activation (Figure 4A). Moreover, only transient, but not sustained, JNK activation was observed in Jurkat cells treated with TRAIL, and NAC pretreatment had little affect on the pattern of TRAIL-induced JNK activation (Figure 4B). These data suggest that AD5-10-induced ROS production within the cell results in sustained JNK activation.

JNK deficiency inhibits AD5-10-induced cell death

To further confirm the role of JNK in AD5-10-induced

B
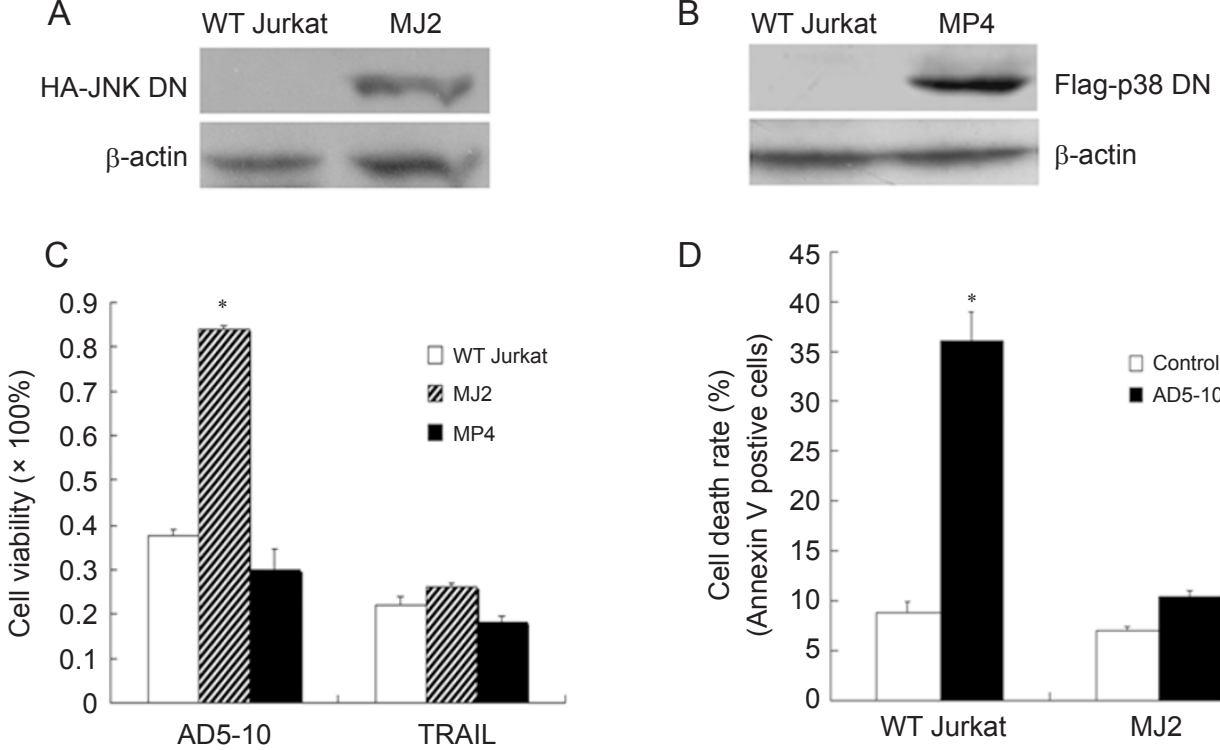

D

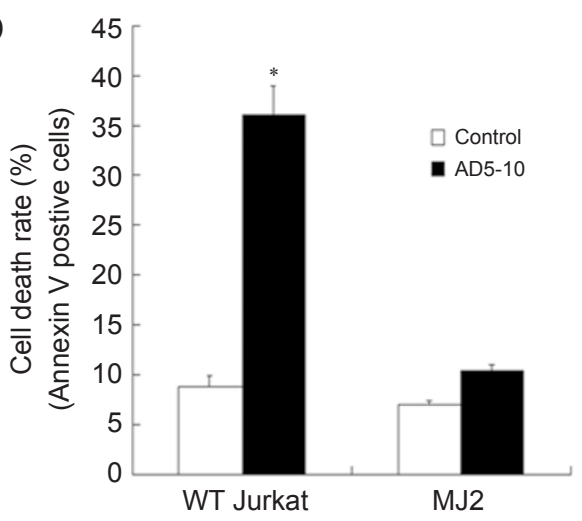

Figure 5 AD5-10-induced cell death is inhibited by JNK deficiency. Wild type Jurkat cells (WT Jurkat) and cells stably transfected with HA-JNK DN (MJ2) (A) or Flag-p38 DN (MP4) (B) were treated with AD5-10 (40 ng/ml) or TRAIL (40 ng/ml) for $4 \mathrm{~h}$. Cell viability $(\mathbf{C})$ was tested by the MTS assay. Apoptosis was analyzed by Annexin-V-FITC and PI staining, followed by flow cytometry (D). 
cell death, Jurkat cells were transfected with vectors expressing HA-JNK dominant-negative mutant (DN) or FLAG-p38 DN. The resultant stable cell lines, MJ2 with HA-JNK DN (Figure 5A) and MP4 with FLAG-p38 DN (Figure $5 \mathrm{~B})$, were treated with AD5-10 $(40 \mathrm{ng} / \mathrm{ml})$ or TRAIL (40 $\mathrm{ng} / \mathrm{ml}$ ) or left untreated for $4 \mathrm{~h}$. Cell viability and the rate of apoptosis were measured by MTS and Annexin-V/PI staining followed by flow cytometry, respectively. As shown in Figure 5C and 5D, cell viability of MJ2 cells (deficient in JNK activity) was remarkably increased. JNK deficiency also significantly reduced AD5-10-induced cell death, whereas p38 deficiency had no effect. However, wild-type (WT) Jurkat, MJ2, and MP4 cells all showed similar sensitivities to TRAIL. These data suggest that JNK, but not $\mathrm{p} 38$, participates in the process of AD5-10-induced cell death.

\section{JNK deficiency inhibits AD5-10-induced mitochondrial dysfunction}

To further evaluate the role of JNK in AD5-10-induced cell death, wild-type Jurkat and MJ2 cells were treated with AD5-10 or left untreated for $4 \mathrm{~h}$, and mitochondrial membrane potential, cardiolipin oxidation, and Endo G and AIF translocation were assayed. As shown in Figure 6, AD5-10 induced more green fluorescence of JC-1 (Figure 6A) in wild-type Jurkat cells than that in MJ2 cells. Primary cardiolipin levels (Figure 6B) in MJ2 cells remained the same as in controls, indicating that JNK deficiency inhibited AD5-10-induced loss of mitochondrial membrane potential and rapid oxidation of cardiolipin. Furthermore, western blot analysis showed that Endo G levels (Figure 6C) in the cytosolic fraction of MJ2 cells treated with AD5-10 were much less than those in wildtype Jurkat cells. This analysis suggests that JNK deficiency inhibited the translocation of Endo G from mitochondria to the cytosol, whereas there was no remarkable difference in AIF translocation (Figure 6D). Quantitative data reinforce this distinction. These data illustrate that JNK deficiency inhibits AD5-10-induced mitochondrial dysfunction.
A

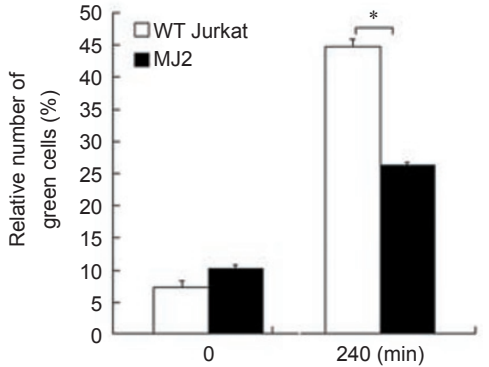

C

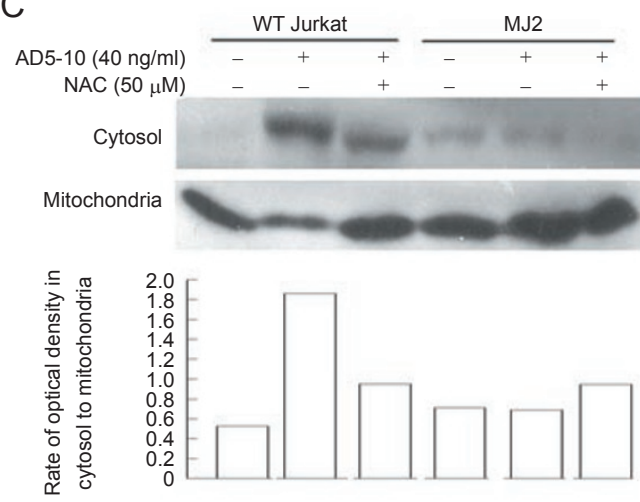

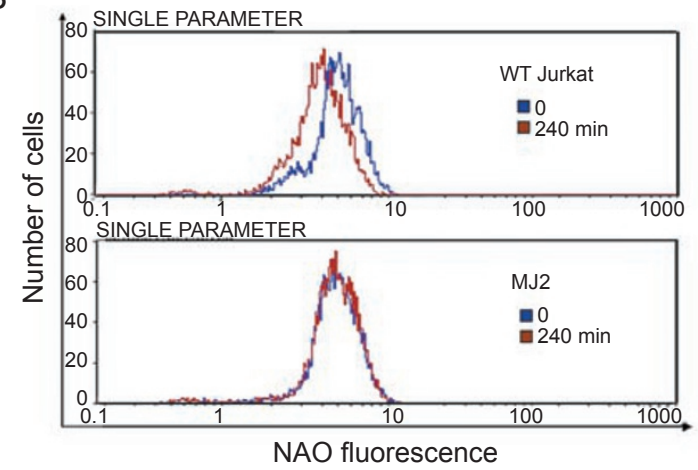

$\mathrm{D}$

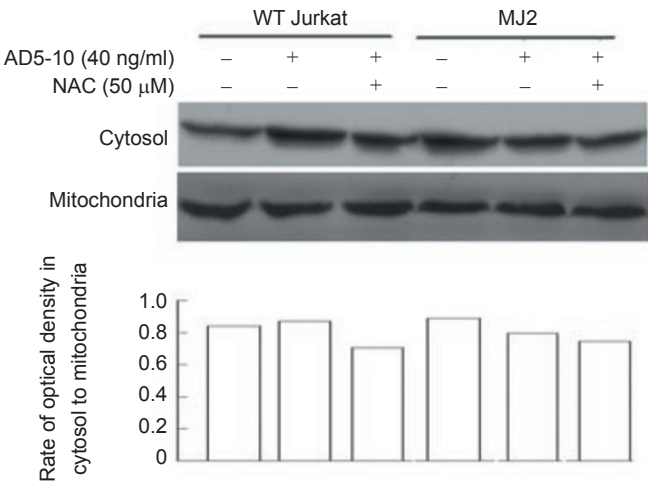

Figure 6 AD5-10-induced mitochondrial damage is inhibited by JNK deficiency. MJ2 cells were treated with AD5-10 (40 ng/ $\mathrm{ml}$ ) for $4 \mathrm{~h}$. Wild type Jurkat cells were used as a control. Mitochondrial membrane potential $(\Delta \psi \mathrm{m})$ was determined with JC-1 $(40 \mathrm{nM})$ staining (A), and oxidative damage to mitochondria was evaluated by NAO staining (B), followed by flow cytometry. Mitochondrial and cytosolic fractions were isolated from the cells treated with AD5-10 (40 ng/ml) in the absence or presence of $50 \mu \mathrm{M}$ NAC for $4 \mathrm{~h}$ and subjected to immunoblot analysis to determine the level of Endo G (C) and AIF (D) by specific antibodies. Quantitative data obtained are included. 


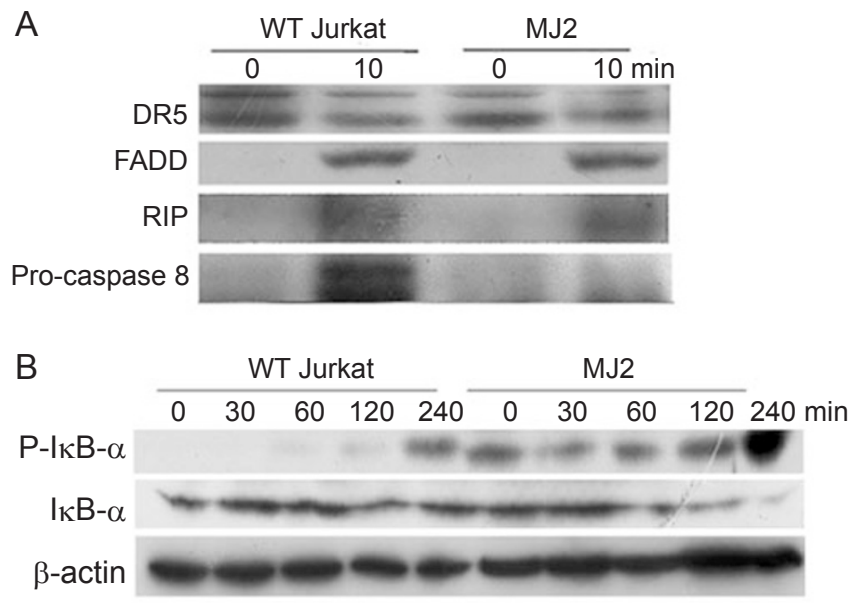

Figure 7 JNK deficiency reduces the recruitment of caspase-8 in DISCs and enhances I $\mathrm{B}$ phosphorylation in Jurkat cells. MJ2 cells were treated with AD5-10 $(40 \mathrm{ng} / \mathrm{ml})$ for the indicated timepoints. Wild type Jurkat cells were used as a control. The recruitment of DR5, caspase-8, FADD and RIP as DISC components $(A)$ and $I_{\kappa} B$ phosphorylation $(B)$ were detected by western blot assays using the indicated specific antibodies.

$J N K$ deficiency decreases the recruitment of caspase-8 to DISCs and increases I $\kappa$ phosphorylation

In order to elucidate the molecular components involved in AD5-10-triggered signaling through DR5, death-inducing signaling complexes (DISCs) recruited by DR5 in wild type Jurkat and MJ2 cells were co-immunoprecipitated from cell lysates after AD5-10 treatment and analyzed by western blot. In MJ2 cells, AD5-10 induced assembly of DISCs containing DR5, FADD, and RIP, but not caspase-8, which is normally part of this complex in wild-type Jurkat cells (Figure 7A). This finding suggests that JNK deficiency inhibits the recruitment of caspase-8 to FADD and partially explains the resistance of MJ2 cells to AD5-10 treatment.

Activated NF- $\kappa \mathrm{B}$ serves as an anti-apoptotic factor by upregulating the expression of caspase inhibitory proteins, and death receptor signaling often involves JNK phosphorylation and NF- $\kappa \mathrm{B}$ activation $[29,30]$. To determine whether NF- $\mathrm{KB}$ is involved in the reduced AD510 sensitivity in JNK-deficient cells, wild-type Jurkat and MJ2 cells were treated with AD5-10 or left untreated for various times, and $\mathrm{NF}-\kappa \mathrm{B}$ activation, represented by I $\mathrm{B}$ phosphorylation, was detected by western blot. As shown in Figure $7 \mathrm{~B}, \mathrm{I}_{\kappa} \mathrm{B}$ was phosphorylated in a timedependent manner by AD5-10 in both MJ2 and wild-type Jurkat cells. Basal phosphorylation of I $\kappa$ B was markedly increased in JNK-deficient MJ2 cells, providing strong support for the idea that NF- $\mathrm{BB}$ was highly activated in the cells, leading to resistance to AD5-10 treatment.

\section{Discussion}

TRAIL and agonistic monoclonal antibodies (mAbs) specific for human DR4 or DR5 selectively kill tumor cells both in vitro and in vivo. This effect occurs without cytotoxicity to most normal cells, which makes these molecules attractive candidates for cancer therapy. The anti-human DR5 monoclonal antibody AD5-10 possesses a strong tumoricidal activity, without toxicity to normal tissues and cells. AD5-10 binds specifically to DR5 without competition with TRAIL and triggers both caspasedependent and -independent signaling pathways [2].

In the present study, we found that, unlike TRAIL and other reported agonistic antibodies to DR5 [31, 32], AD5-10 induces ROS production and decrease of GSH and GSSG content in a time-dependent manner in Jurkat leukemia cells. Addition of ROS scavengers such as GSH or the antioxidant NAC effectively suppresses AD5-10triggered cell death, loss of mitochondrial membrane potential, and oxidation of cardiolipin in the mitochondrial membrane, indicating that redox state plays a critical role in the cell death process. Physiologically, ROS and GSH work to keep a redox balance, which maintains an environment conducive to cell survival [33, 34]. However, overproduction of ROS or lack of GSH may rapidly damage the normal function of mitochondria, leading to cell death. We have demonstrated that activation of DR5 alone is able to mediate overproduction of ROS and a corresponding decrease in GSH (Figure 1). Because Jurkat T-lymphocytes lack conventional NADPH oxidase enzymes, which are used for oxidative bursts in granulocytes, increased demand for energy production due to increased cellular respiration is likely responsible for higher superoxide levels in these cells [35].

JNK and p38 can be activated under diverse environmental stress conditions in various cell types. Prior studies on receptor-mediated JNK and p38 activation mostly focused on the TNF receptor superfamily, especially that of $\mathrm{TNF} \alpha$ [9-14]. $\mathrm{TNF} \alpha$ receptor-mediated JNK and p38 activation is generally rapid and transient, reaching a peak after approximately $10 \mathrm{~min}$ and then declining to basal levels in MEF or DKO cells. In NF$\kappa \mathrm{B}$ downregulated cells, JNK and p38 are constitutively active, although their activity can be inhibited by the antioxidant butylated hydroxy anisole (BHA), but not the pan-caspase inhibitor Z-VAD [11]. Wicovsky et al. [36] reported that JNK induces caspase activity in response to $\mathrm{TNF} \alpha$ in a cell type-specific manner. We have shown in the present study that AD5-10 induces transient JNK and p38 activation within $15 \mathrm{~min}$. Sustained JNK activation, but not p38 activation, was observed $2 \mathrm{~h}$ later (Figure $4 \mathrm{~A}$ ), when NF-kB was also activated. These results were 
in contrast with reports by Sakon et al. [10] and Deng et al. [11], which showed that $\mathrm{TNF} \alpha$ and Fas were only able to mediate ROS production and sustained JNK activation if $\mathrm{NF}-\mathrm{kB}$ was inhibited. It is possible that the activation of NF- $\mathrm{NB}$ induced by DR5 was not strong enough to affect JNK phosphorylation, because inhibition of $\mathrm{NF}-\kappa \mathrm{B}$ activation by Leu-Leu-Norleucinal (LLNL) did enhance JNK activation (data not shown). Alternatively, differences in cell type, stimuli, or even specific intracellular conditions may have caused these discrepancies. $\mathrm{NAC}$, an antioxidant, completely inhibits long-term JNK activation but has no effect on the transient activation of JNK or p38 in Jurkat cells, results which are identical to those reported by Deng et al. [11].

The involvement of JNK in receptor-mediated programmed cell death remains controversial [37, 38]. Previous evidence suggests that transient JNK activation is associated with gene expression during cell proliferation or differentlation, whereas sustained JNK activation may promote cell death in cell type- and stimulus-dependent manners $[10,36]$. First, JNK phosphorylation activates the transcription factor AP-1, which translocates to the nucleus to regulate the expression of various genes, such as FasL, p53, c-myc, and bcl-2 family members. Second, ROS are specifically detected in mitochondria or submitochondrial particles [39], inducing the oxidation of mitochondrial proteins. JNK activation by ROS further induces the loss of mitochondrial membrane potential, phosphorylation of downstream proteins such as Bim, Bcl-xl and Bcl-2, and cleavage of Bid [39, 40], leading to subsequent release of various proapoptotic factors. Recently, Zhou et al. [41] provided evidence that phosphorylated JNK translocates to mitochondria and subsequently phosphorylates the E1 $\alpha$ subunit of pyruvate dehydrogenase. Phosphorlyation leads to a decline in pyruvate dehydrogenase activity and, consequently, a shift to anaerobic pyruvate metabolism, leading to necrosis.

Endo G and AIF are two inducing signals that have been implicated in non-apoptotic or necrotic programmed cell death (PCD), because they can be translocated into the nucleus and induce DNA fragmentation $[30,42]$. We have shown that both AD5-10 and TRAIL induce AIF and Endo $G$ release from mitochondria into the cytosol (Figure 3D). NAC blocks the release of additional AIF and Endo $G$ into the cytoplasm and the translocation of Endo G into the nucleus in AD5-10-treated cells (Figure 3D-3F). We speculate that ROS-dependent Endo G translocation is responsible for the downstream DNA fragmentation seen in AD5-10-induced PCD. Furthermore, stable Jurkat cells expressing a dominant-negative form of JNK exhibit less sensitivity to AD5-10, which is consistent with reduced damage to mitochondria and less translocation of Endo G (Figure 6A-6C). In addition, basal $\mathrm{I}_{\kappa} \mathrm{B}$ phosphorylation is markedly increased in JNK-deficient cells. Activated NF- $\kappa \mathrm{B}$ may upregulate the caspase-inhibitory proteins FLIP, IAPs, and Bcl-2 [43], which may further influence the recruitment of subunits to DISCs, accounting for the reduced recruitment of caspase-8 in our assay (Figure 7A). All these data help explain the reduced sensitivity of MJ2 cells to AD5-10 and provide further evidence that JNK is closely involved in AD5-10-induced cell death.

It is interesting to note that AD5-10-induced cell death is different from that induced by TRAIL in terms of ROS production and JNK activation. However, the mechanisms behind these differences remain to be determined. We speculate that different agonists are able to bind their receptors by distinct mechanisms, resulting in the recruitment of different components to the DISC. Several observations support this hypothesis. First, AD510 and TRAIL do not compete for binding to DR5, indicating that they have different binding sites [2]. Second, the affinity of AD5-10 for DR5 is much higher than that of TRAIL for DR5 [2]. Third, TRAIL and DR5 act as trimers, whereas antibodies exist as dimers. The differing oligomerization states of ligands and their receptors may affect the configuration of the receptors, resulting in different signals. Park et al. [32] has shown that an antibody against DR5 triggered recruitment of TRAF2 and TRADD, but not of caspase- 8 or FADD, leading to autophagy. Further investigation is needed to explore the differences between AD5-10- and TRAIL-induced apoptosis.

Although there is no firm correlation between specific cellular proteins and apoptosis, because PCD involves diverse and complex processes, it is now evident that multiple pathways lead to cell death, depending on stimulus and cell type $[44,45]$. We have demonstrated that overproduction of ROS induces secondary JNK activation, and plays a critical role in AD5-10-induced cell death. How AD5-10 (but not TRAIL) induces ROS accumulation and how JNK impairs the function of mitochondria remain to be investigated. Because DR5 is expressed in most cancer cells and is closely associated with tumorigenicity and chemosensitivity, further study of its signaling pathways will provide additional information about the mechanisms of PCD and may outline new strategies for leukemia and solid tumor therapies.

\section{Materials and Methods}

\section{Cell lines, reagents, and antibodies}

Jurkat and HCT116 cells were purchased from the American Type Culture Collection (ATCC, Rockville, MD, USA). Cells were cultured in RPMI 1640 medium (Invitrogen, CA, USA), 
supplemented with $10 \%$ heat-inactivated fetal calf serum (Hyclone, Logan, UT, USA), penicillin (100 U/ml), and streptomycin $(100 \mu \mathrm{g} / \mathrm{ml})$ at $37{ }^{\circ} \mathrm{C}$ in a humidified atmosphere of $5 \% \mathrm{CO}_{2}$. JNK and p38 dominant-negative plasmids were kindly provided by Dr Shimin Hu (University of Pennsylvania). Recombinant human TRAIL (residues 114-281) without any epitope tag was purchased from KOMA Biotech (Shenzhen, China). AD5-10 was prepared as previously described by Guo et al. [2]. Reduced glutathione (GSH) was purchased from Promega (Madison, WI). G418 was obtained from Alexis (Lausen, Switzerland). Anti-caspase-8 and anti-Endo $\mathrm{G}$ antibodies were purchased from Calbiochem (La Jolla, CA). Anti-Bcl-2, anti-Bax, anti-Bik, anti-Bad, anti-JNK, anti-p38, and anti-I $\mathrm{K}$ B antibodies were purchased from Santa Cruz (CA, USA). Antibodies against the phosphorylated forms of p38 (p-38), JNK (p-JNK) and $\mathrm{I} \kappa \mathrm{B}(\mathrm{p}-\mathrm{I} \kappa \mathrm{B})$ were purchased from Cell Signaling Technology, Inc. (Beverly, MA). Anti-PARP, anti-actin and Nacetylcysteine (NAC) were purchased from Sigma (CA, USA). Horseradish peroxidase (HRP)-conjugated anti-mouse IgG, antirabbit IgG and anti-goat IgG were purchased from ZhongShan Co. (Beijing, China).

\section{Cell viability assay}

Jurkat cells were seeded in 96-well plates $\left(4 \times 10^{4}\right.$ cells/well $)$ and preincubated with NAC or GSH or left untreated for $30 \mathrm{~min}$ before the addition of other stimulants. After the desired time, cell viability was measured by the CellTiter 96 AQueous nonradioactive kit (MTS, Promega, Madison, WI), according to the manufacturer's instructions.

\section{Cell death assay}

Treated cells were stained using an Annexin V/PI kit, according to the manufacturer's instructions (BD Biosciences Clontech, NY). The cells were harvested, washed once with cold phosphatebuffered saline (PBS) and stained with $200 \mu \mathrm{l}$ of binding buffer containing Annexin-V-FITC for $30 \mathrm{~min}$ on ice. PI was added to the buffer 5 min later, then $300 \mu l$ binding buffer were added, and cells were assayed by flow cytometry (FACScan, Becton Dickinson, Germany).

\section{Measurement of ROS}

ROS levels were measured in live cells by an ROS detection kit from BiYunTian Co. (Jiangsu, China). Cells $\left(1 \times 10^{6} / \mathrm{ml}\right)$ were incubated in complete medium containing $4 \mu \mathrm{M}$ DCFA for $20 \mathrm{~min}$ at $25^{\circ} \mathrm{C}$, washed twice and then resuspended in complete medium. Finally, DCF fluorescence in live cells was detected by flow cytometry (FACScan, Becton Dickinson, Germany).

\section{Measurement of intracellular GSH}

Intracellular GSH level was measured by a GSH and GSSG assay kit from BiYunTian Co. (Jiangsu, China). Briefly, treated cells were harvested, washed twice with PBS and then lysed in lysis buffer. The lysate was assayed for GSH and GSSG levels by CellTiter 96 AQueous, according to the manufacturer's instructions.

\section{Mitochondrial membrane potential}

Loss of mitochondrial membrane potential in cells was detected by the mitochondrial selective dye JC-1 (Molecular Probes, Eugene, OR). Treated Jurkat cells were harvested, suspended in $1 \mathrm{ml}$ of complete medium containing $10 \mu \mathrm{g} \mathrm{JC}-1$ at $25^{\circ} \mathrm{C}$ for $20 \mathrm{~min}$, and washed twice with culture medium. Cell pellets were then resuspended in $0.5 \mathrm{ml}$ complete medium and subjected to flow cytometry (FACScan, Becton Dickinson, Germany).

\section{Western blot analysis}

Treated cells were washed once with PBS and lysed in lysis buffer. The lysates were subjected to SDS-PAGE, and the proteins in the gel were subsequently electrotransferred to a polyvinylidene difluoride (PVDF) membrane (Millipore, Boston). After being blocked with 5\% nonfat dry milk in TBS-T for $1-2 \mathrm{~h}$ at room temperature, the PVDF membrane was incubated at $4{ }^{\circ} \mathrm{C}$ overnight with the indicated primary antibody, diluted in TBS-T containing $5 \%$ nonfat dry milk. The membrane was then washed three times with TBS-T and probed with HRP-conjugated secondary antibodies at room temperature for 1.5-2 $\mathrm{h}$. After being washed four times with TBS-T, proteins were visualized with the ECL system (Millipore, Boston).

\section{Preparation of cytosolic and mitochondrial fractions}

Cytosolic and mitochondrial fractions were prepared as described [46]. Cells were harvested, washed twice with cold PBS and then incubated overnight at $4{ }^{\circ} \mathrm{C}$ with $1.0 \mathrm{ml}$ cold lysis buffer containing $20 \mathrm{mM}$ HEPES-KOH (pH 7.5), $10 \mathrm{mM} \mathrm{KCl,} 1.5 \mathrm{mM}$ $\mathrm{MgCl}_{2}, 1 \mathrm{mM}$ sodium EDTA, $1 \mathrm{mM}$ sodium EGTA, $250 \mathrm{mM}$ sucrose, $5 \mu \mathrm{g} / \mathrm{ml}$ pepstatin A, $10 \mu \mathrm{g} / \mathrm{ml}$ leupeptin and $2 \mu \mathrm{g} / \mathrm{ml}$ aprotinin. The lysates were centrifuged at $1000 \mathrm{rpm}$ for $5 \mathrm{~min}$, after which supernatants were centrifuged at $39000 \mathrm{rpm}$ for $30 \mathrm{~min}$, and the pellets were re-suspended in $30 \mu 1$ lysis buffer. Samples in $1 \times$ SDS loading buffer were boiled for $5 \mathrm{~min}$ and then loaded on a $12 \%$ polyacrylamide gel for SDS-PAGE.

\section{Electro-transfection}

Jurkat cells were transfected with Flag-pcDNA3-P38-DN or HA-pcDNA3-JNK-DN using the Jurkat nucleofector kit (Amaxa), according to the manufacturer's instructions. The cells were seeded in 6-well plates in complete medium, and G418 (800 $\mu \mathrm{g} /$ $\mathrm{ml}$ ) was added after $24 \mathrm{~h}$ of culture. G418-resistant clones were isolated about 20 days later and then used in further experiments.

\section{Confocal laser microscopic analysis}

Endo $\mathrm{G}$ release was observed by confocal laser microscopy. Cells were fixed on slides with 4\% paraformaldehyde in PBS. Cells were incubated with 5\% nonfat dry milk in PBS-T for $1 \mathrm{~h}$ at room temperature and then incubated at $4{ }^{\circ} \mathrm{C}$ overnight with the indicated primary antibody, diluted in PBS-T containing 5\% nonfat dry milk. After being washed three times with PBS-T, the cells were probed with FITC-conjugated secondary antibodies at room temperature for $2 \mathrm{~h}$. After three washes, the cells were stained with 4',6-diamidino-2-phenylindole (DAPI) for 5min. Slides were observed under a confocal laser-scanning microscope (LSM 510UV/ META, Carl Zeiss Microimaging, Thomwood, New York).

\section{Immunoprecipitation}

Cells $\left(3 \times 10^{7}\right)$ were incubated with or without AD5-10 for 5 min, harvested and washed twice with cold PBS. Next, the cell pellets were lysed in $1 \mathrm{ml}$ ice-cold lysis buffer $(50 \mathrm{mM}$ Tris- $\mathrm{HCl}$, pH 7.5, $150 \mathrm{mM} \mathrm{NaCl}, 10 \%$ glycerol, $10 \mathrm{mM} \mathrm{NaF}, 1 \% \mathrm{NP}-40,5$ $\mu \mathrm{g} / \mathrm{ml}$ pepstatin A, $10 \mu \mathrm{g} / \mathrm{ml}$ leupeptin and $2 \mu \mathrm{g} / \mathrm{ml}$ aprotinin) for 
$30 \mathrm{~min}$. Cell debris was pelleted by centrifugation for $10 \mathrm{~min}$ at $13000 \mathrm{rpm}$, and immune complexes were precipitated using $30 \mu \mathrm{l}$ of protein A Sepharose (Sigma) for $2 \mathrm{~h}$ at $4{ }^{\circ} \mathrm{C}$. After centrifugation and three washes in lysis buffer, immunoprecipitated material was suspended in $1 \times$ SDS-loading buffer, boiled for $5 \mathrm{~min}$, and resolved on SDS-PAGE.

\section{Statistical analysis}

All data were expressed as mean values \pm standard deviation (s.d.), and Student's $t$ test was used for evaluating statistical significance. $P$ values were considered statistically significant when less than 0.05 .

\section{Acknowledgments}

We thank Dr Shimin Hu for his generous gifts of constructs. This work was partially supported by National Natural Science Foundation of China (Grant Nos. 30571687 and 30721063 ) and by State Key Basic Research Program of China (Grant No. 2007CB507404).

\section{References}

1 Ashkenazi A. Targeting death and decoy receptors of the tumour-necrosis factor superfamily. Nat Rev Cancer 2002; 2:420-430.

2 Guo Y, Chen C, Zheng Y, et al. A novel anti-human DR5 monoclonal antibody with tumoricidal activity induces caspase-dependent and caspase-independent cell death. J Biol Chem 2005; 280:41940-41952.

3 Schulze-Osthoff K, Ferrari D, Los M, Wesselborg S, Peter ME. Apoptosis signaling by death receptors. Eur J Biochem 1998; 254:439-459.

4 Bodmer JL, Holler N, Reynard S, et al. TRAIL receptor-2 signals apoptosis through FADD and caspase-8. Nat Cell Biol 2000; 2:241-243.

5 Wang WH, Gregori G, Hullinger RL, Andrisani OM. Sustained activation of p38 mitogen-activated protein kinase and c-Jun N-terminal kinase pathways by hepatitis B virus X protein mediates apoptosis via induction of Fas/FasL and tumor necrosis factor (TNF) receptor $1 / \mathrm{TNF} \alpha$ expression. Mol cell Biol 2004; 24:10352-10365.

6 Nakshatri H, Rice SE, Bhat-Nakshatri P. Antitumor agent parthenolide reverses resistance of breast cancer cells to tumor necrosis factor-related apoptosis-inducing ligand through sustained activation of c-Jun N-terminal kinase. Oncogene 2004; 23:7330-7344.

7 Eliopoulos AG, Gallagher NJ, Blake SM, Dawson CW, Young LS. Activation of the p38 mitogen-activated protein kinase pathway by Epstein-Barr virus-encoded latent membrane protein 1 coregulates interleukin-6 and interleukin-8 production. J Biol Chem 1999; 274:16085-16096.

8 Zhang Y, Chen F. Reactive oxygen species (ROS), troublemakers between nuclear factor-kappaB (NF-kappaB) and cJun NH2 terminal kinase (JNK). Cancer Res 2004; 64:19021905.

9 Kamata H, Honda S, Maeda S, Chang L, Hirata H, Karin M. Reactive oxygen species promote TNFalpha-induced death and sustained JNK activation by inhibiting MAP kinase phos- phatases. Cell 2005; 120:649-661.

10 Sakon S, Xue X, Takekawa M, et al. NF-kappaB inhibits TNF-induced accumulation of ROS that mediate prolonged MAPK activation and necrotic cell death. EMBO J 2003; 22:3898-3909.

11 Deng Y, Ren X, Yang L, Lin Y, Wu X. A JNK-dependent pathway is required for TNFalpha-induced apoptosis. Cell 2003; 115:61-70.

12 Wang L, Azad N, Kongkaneramit L, et al. The Fas death signaling pathway connecting reactive oxygen species generation and FLICE inhibitory protein down-regulation. J Immunol 2008; 180:3072-3080.

13 Patel NS, Chatterjee PK, Di Paola R, et al. Endogenous interleukin-6 enhances the renal injury, dysfunction and inflammation caused by ischemia/reperfusion. J Pharmacol Exp Ther 2005; 312:1170-1178.

14 Ventura JJ, Cogswell P, Flavell RA, Baldwin AS Jr, Davis RJ. JNK potentiates TNF-stimulated necrosis by increasing the production of cytotoxic reactive oxygen species. Gene Dev 2004; 18:2905-2915.

15 Shiraishi T, Yoshida T, Nakata S, et al. Tunicamycin enhances tumor necrosis factor-related apoptosis-inducing ligandinduced apoptosis in human prostate cancer cells. Cancer Res 2005; 65:6364-6370.

16 Ohtsuka T, Zhou T. Bisindolylmaleimide VIII enhances DR5 mediated apoptosis through the MKK4/JNK/p38 kinase and the mitochondrial pathways. J Biol Chem 2002; 277:2929429303.

17 Holler N, Zaru R, Micheau O, et al. Fas triggers an alternative, caspase- 8 independent cell death pathway using the kinase RIP as effector molecule. Nat Immunol 2000; 1:489-495.

18 Kim EH, Yoon M, Kim SU, Kwon TK, Sohn S, Choi KS. Arsenic trioxide sensitizes human glioma cells, but not normal astrocytes, to TRAIL-induced apoptosis via CCAAT/ enhancer-binding protein homologous protein-dependent DR5 up-regulation. Cancer Res 2008; 68:266-275.

19 Efferth T, Giaisi M, Merling A, Krammer PH, Li-Weber M. Artesunate induces ROS-mediated apoptosis in doxorubicinresistant T leukemia cells. PLoS One 2007; 2: e693. DOI: 10.1371/journal.pone.0000693

20 Shankar S, Ganapathy S, Srivastava RK. Sulforaphane enhances the therapeutic potential of TRAIL in prostate cancer orthotopic model through regulation of apoptosis, metastasis, and angiogenesis. Clin Cancer Res 2008; 14:6855-6866.

21 Kim S, Lee TJ, Leem J, Choi KS, Park JW, Kwon TK. Sanguinarine-induced apoptosis: generation of ROS, down-regulation of Bcl-2, c-FLIP, and synergy with TRAIL. $J$ Cell Biochem 2008; 104:895-907.

22 Yi J, Yang J, He R, et al. Emodin enhances arsenic trioxideinduced apoptosis via generation of reactive oxygen species and inhibition of survival signaling. Cancer Res 2004; 64:108-116.

23 Herrera B, Alvarez AM, Sánchez A, et al. Reactive oxygen species (ROS) mediates the mitochondrial-dependent apoptosis induced by transforming growth factor (beta) in fetal hepatocytes. FASEB J 2001; 15:741-751.

24 Gelinas C, White E. BH3-only proteins in control: specificity regulates MCL-1 and BAK-mediated apoptosis. Genes Dev 2005; 19:1263-1268. 
25 Das S, Khan N, Mukherjee S, et al. Redox regulation of resveratrol-mediated switching of death signal into survival signal. Free Radic Biol Med 2008; 44:82-90.

26 Atlante A, Calissano P, Bobba A, Azzariti A, Marra E, Passarella $\mathrm{S}$. Cytochrome $\mathrm{c}$ is released from mitochondria in a reactive oxygen species (ROS)-dependent fashion and can operate as a ROS scavenger and as a respiratorysubstrate incerebellar neurons undergoing excitotoxic death. $J$ Biol Chem 2000; 275:37159-37166.

27 Park MT, Kim MJ, Kang YH, et al. Phytosphingosine in combination with ionizing radiation enhances apoptotic cell death in radiation-resistant cancer cells through ROS-dependent and -independent AIF release. Blood 2005; 105:1724-1733.

28 Hamada M, Sumi T, Iwai S, Nakazawa M, Yura Y. Induction of endonuclease G-mediated apopotosis in human oral squamous cell carcinoma cells by protein kinase $\mathrm{C}$ inhibitor safingo. Apoptosis 2006; 11:47-56.

29 Varfolomeev E, Maecker H, Sharp D, et al. Molecular determinants of kinase pathway activation by Apo2 ligand/tumor necrosis factor-related apoptosis-inducing ligand. $\mathrm{J} \mathrm{Biol} \mathrm{Chem}$ 2005; 280:40599-40608.

30 Nakano H, Nakajima A, Sakon-Komazawa S, Piao JH, Xue X, Okumura K. Reactive oxygen species mediate crosstalk between NF-kappaB and JNK. Cell Death Differ 2006; 13:730737.

31 Ichikawa K, Liu W, Zhao L, et al. Tumoricidal activity of a novel anti-human DR5 monoclonal antibody without hepatocyte cytotoxicity. Nat Med 2001; 7:954-960.

32 Park KJ, Lee SH, Kim TI, et al. A human scFv antibody against TRAIL receptor 2 induces autophagic cell death in both TRAIL-sensitive and TRAIL-resistant cancer cells. Cancer Res 2007; 67:7327-7334.

33 Ueda S, Masutani H, Nakamura H, Tanaka T, Ueno M, Yodoi J. Redox control of cell death. Antioxid Redox Sign 2002; 4:405414.

34 Hancock JT, Desikan R, Neill SJ. Does the redox status of cytochrome $\mathrm{c}$ act as a fail-safe mechanism in the regulation of programmed cell death? Free Radic Biol Med 2001; 31:697-
703.

35 Hildeman DA, Mitchell T, Kappler J, Marrack P. T cell apoptosis and reactive oxygen species. J Clin Invest 2003; 111:575-581.

36 Wicovsky A, Müller N, Daryab N, et al. Sustained JNK activation in response to tumor necrosis factor is mediated by caspases in a cell type-specific manner. J Biol Chem 2007; 282:2174-2183.

37 Kischkel FC, Hellbardt S, Behrmann I, et al. Cytotoxicity-dependent APO-1 (Fas/CD95)-associated proteins form a deathinducing signaling complex (DISC) with the receptor. $E M B O$ $J$ 1995; 14:5579-5588.

38 Zong WX, Thompson CB. Necrotic death as a cell fate. Gene Dev 2006; 20:1-15

39 Nagata K, Iwasaki Y, Yamada T, et al. Overexpression of manganese superoxide dismutase by $\mathrm{N}$-acetylcysteine in hyperoxic lung injury. Respir Med 2007; 101:800-807.

40 Liu J, Lin A. Role of JNK activation in apoptosis: a doubleedged sword. Cell Res 2005; 15:36-42.

41 Zhou Q, Lam PY, Han D, Cadenas E. c-Jun N-terminal kinase regulates mitochondrial bioenergetics by modulating pyruvate dehydrogenase activity in primary cortical neurons. J Neurochem 2008; 104:325-335.

42 Li LY, Luo X, Wang X. Endonuclease G is an apoptotic DNase when released from mitochondria. Nature 2001; 412:9599.

43 Kelekar A, Thompson CB. Bcl-2-family proteins: the role of the BH3 domain in apoptosis. Trends Cell Boil 1998; 8:324330.

44 Nelson DA, White E. Exploiting different ways to die. Gene Dev 2004; 18:1223-1226

45 Fiers W, Beyaert R, Declercq W, Vandenabeele P. More than one way to die: apoptosis, necrosis and reactive oxygen damage. Oncogene 1999; 18:7719-7730.

46 Wang M, Liu Y, Liu S, Zheng D. 8-Chloro-adenosine sensitizes a human hepatoma cell line to TRAIL-induced apoptosis by caspase-dependent and -independent pathways. Oncol Rep 2004; 12:193-199. 